\title{
Organ transplantation logistics: a case for Turkey
}

\author{
Sinem Savaşer ${ }^{1}$. Ömer Burak Kınay ${ }^{2}$. Bahar Yetis Kara ${ }^{3}$. Pelin Cay ${ }^{4}$ \\ Received: 26 January 2018 / Accepted: 2 November 2018 \\ (c) Springer-Verlag GmbH Germany, part of Springer Nature 2018
}

\begin{abstract}
Logistics is one of the key elements of organ transplantation operations. In this study, maximizing the potential compatible donor-recipient matches within the cold ischemia time bounds (duration that an organ can survive without blood supply) is the main problem that is addressed. While addressing this problem, the effects of clustering structures on the potential organ matches are investigated. We analyze Turkey's organ transplantation logistics structure based on its dynamics and provide new mathematical models for maximizing potential-weighted intra-regional organ transplantation flow via evaluating different types of transportation modes while meeting the specified time bounds. This approach considers only maximizing the potential flow within a single time bound, so that it may not perform effectively for every organ type. To remedy this situation, another mathematical model that maximizes the potential flow of multiple organ types has also been developed. Additionally, in order to evaluate the performance of our results, using the outputs of the deterministic mathematical models, we developed a simulation model to mimic the uncertain environment realistically while being able to model the components of hierarchical systems. Extensive computational analysis using a variety of performance measures has revealed that Turkey's organ transplantation network can be improved by re-clustering.
\end{abstract}

Keywords OR in health services · Organ transplantation logistics · Location · Hierarchical allocation

S. Savaşer, Ö. B. Kınay: This research was initiated when the author was at Department of Industrial Engineering, Bilkent University, Ankara, Turkey.

$凶 \quad$ Bahar Yetis Kara

bkara@bilkent.edu.tr

1 Department of Operations Management, Joseph L. Rotman School of Management, University of Toronto, Toronto, ON, Canada

2 Department of Management Sciences, University of Waterloo, Waterloo, ON, Canada

3 Department of Industrial Engineering, Bilkent University, Ankara, Turkey

4 Department of Industrial and Systems Engineering, Lehigh University, Bethlehem, PA, USA 


\section{Introduction}

Organ transplantation is a complex treatment method in medicine. The major difference that separates this treatment from others is that this method requires a donor (a living or dead person who donates his/her organ(s)) and a recipient (a patient who needs the donated organ in order to survive or improve his/her quality of life). The procedure can be defined as the transfer of an organ from a donor to a recipient to replace a damaged or absent organ of him/her. Although helpful, the procedure can only be performed on certain organ types including heart, kidneys, liver, lungs, and pancreas and certain tissues such as cornea, skin, heart valves, bones, etc. Kidney transplants are the most common ones followed by liver and heart transplants (Human Organ Transplantation 2016).

Despite the fact that this treatment can save many lives and/or improve the quality of life of many patients, organ transplantations involve obstacles as well. A major problem is the significant gap between organ supply and demand. In the USA alone, 20 people die every day because they cannot access an organ in-time, and more than 110,000 patients are known to be on waiting lists (UNOS 2018). In Turkey, only 4,500 organs were transplanted in 2015 and as of May 2016, 25,500 people were still waiting for a suitable organ (Recent Data 2016).

Another challenge is the compatibility of the donated organ with the recipient. It is not only enough to find just the organ but also there are quite a lot of factors that needed to be satisfied in order to determine a patient as a potential recipient. An organ must be biologically compatible (blood type and tissue match and immune status) with a recipient. Besides this, factors such as age and psychological situation are also important constraints. This makes finding a recipient for an organ difficult as majority of the available donations cannot be used.

Cold ischemia time is the duration that an organ can survive without a blood supply. This limits the time an organ can spend outside of a body, thus reducing the number of potential recipient to those that are in close proximity. Since increasing the time spent without a blood supply decreases the chance of the organ functioning properly in the recipient's body, distance becomes a concern in transplantation success. Hence, this points forward the significance of donated organ's and recipient's locations in the system.

There are some other issues to be considered in this process. For instance, the transplantation surgeries cannot be performed at any hospital; as a matter of fact, these operations can only be done at specialized hospitals using specific equipments with respect to each organ type. Moreover, organ harvesting (taking the organ from donor's body) can only be performed by medical teams specialized in that particular organ. Donors can come from any city, but the recipients are only located in a hospital with an organ transplantation center. This increases the importance of the location of the donor's city.

Transplant rejection is another issue regarding this procedure. Since the organ is foreign to the body, the immune system may attack it despite the immunosuppressants patient takes. This results in organ rejection which requires the immediate removal of the organ from the body. 
All of these complications imply that it is hard to maintain a well-functioning system. Most of these issues cannot be fully controlled; however, the logistics aspect of the network can be improved. Since the locations of patients and hospitals and transportation times are crucial aspects of an organ transplantation network, logistics has a vital role. To address these issues, many researches have been done on the optimal organ transplantation logistics system; in the USA by Stahl et al. (2005), Kong et al. (2010), Demirci et al. (2012) and Gentry et al. (2015), in Italy by Bruni et al. (2006), in Belgium by Beliën et al. (2012) and in Iran by Zahiri et al. (2014b).

There are two major approaches practiced by different countries to find the best recipient for a donated organ in the transplantation system while considering the matching criteria: the centralized method and the hierarchical method. The centralized method entails the use of a single waiting list for an entire region or country. When an organ is donated, the patients obtain a matching score depending on their blood and tissue type, size of the organ, age, level of emergency, time spent on the waiting list and distance between the donor and recipient (OPTN 2016). If the recipient with the highest score is suitable for transplantation, i.e., his/her current health and immune status are fine, or donor's medical history does not cause any problems, then the match is determined. Otherwise, the next highest-scored recipient is selected. This procedure goes on until a successful match is made. In this system, even if there is a patient waiting for an organ in the same hospital, this patient may not necessarily be the recipient. Eurotransplant, which coordinates organ allocation among its seven member countries, is an example of the centralized method (Beliën et al. 2012).

The hierarchical method considers multiple waiting lists within a country, each of which associated with a structural or geographical entity such as a hospital, a city or a region. When an organ is available for transplantation, candidates in the first layer of the hierarchy (e.g., usually the donor's hospital) are evaluated; if there are no suitable matches, then candidates in the second layer of the hierarchy (e.g., the donor's city) are examined. This procedure is repeated at all layers of the hierarchy until a match is found. This system is used by many countries including the USA, Italy and Turkey. Although the method is the same, application varies between countries based on healthcare policies in practice. For instance, in Italy, the donated organ is first taken to the center where the allocation of the organ is made and then taken to the recipient's hospital, while in Turkey and in the USA, the donated organ is directly transferred to the recipient's hospital.

Although the centralized method is more fair as the organ is transferred to the most suitable patient, the hierarchical method ensures that the organ is transferred to the closest suitable recipients, which increases the chances of keeping the organ functional.

This study aims to improve Turkey's hierarchical system by evaluating the effects of clustering structures on the potential matchings for organ transplantation. This is done by exploring the optimal clusters for different transportation options as a strategiclevel decision. The performance of these clusters is measured by a discrete-event simulation model so that the features of the organ transplantation system that cannot be captured by the optimization models (such as hierarchical matching behavior, vehicle availability, emergency cases) can be represented and evaluated. The use of optimization in tandem with simulation models for organ transplantation framework is 
an approach that generates optimum solutions for strategic decisions (such as facility location) while having the chance to observe and evaluate the performance of those solutions before their real-life implementations.

As each organ type has its own characteristics, the logistics network for distinct organs may have different structures. Therefore, this study will discuss regional clusters for each organ type; however, dividing the country into different regions for each organ type is not feasible. Thus, a single clustering network with the best overall performance has to be determined. In order to achieve this, performance of the clusters of a particular organ will be evaluated based on their suitability for other organ types. The most suitable clustering structure will be selected by the decision makers in the Ministry of Health.

The rest of the article is structured as follows: in Sect. 2, a brief analysis of the Turkish organ transplantation system is given. Next, we discuss the extended literature on healthcare logistics and organ transplantation in Operations Research (OR) in Sect. 3. Then, the optimization and simulation models developed in this study are presented in Sect. 4 and that is followed by the computational results based on the Turkish transplantation system in Sect. 5. In Sect. 6, an extended modeling approach is discussed and its numerical results are provided. Finally in Sect. 7, the significant outcomes of the current Turkish organ transplantation system (and ways to improve its performance) are also discussed along with possible future research opportunities.

\section{The case for Turkey}

In Turkey, organ allocations are coordinated by the National Coordination Center (NCC) and nine regional coordination centers (RCCs). The NCC is responsible for the organization's managerial- and strategic-level decisions, while the RCCs coordinate organ transplantation between cities in their region. So, while the RCCs coordinate the region-based searches, the NCC coordinates communication among RCCs. In order to maintain the coordination, each city is assigned to exactly one RCC.

Not every city has organ transplantation-capable hospitals, and each suitable hospital specializes in only one organ type. Even if there may exist a donor in each city, potential recipients are located in only those cities which have transplantation-capable hospital(s). Therefore, each patient is registered in the system via an appropriate hospital, and every organ type has a separate waiting list. Figure 1 depicts the current RCC locations and the assignments of the cities to the RCCs in the Turkish organ allocation system.

Matching a donor with a recipient in Turkey works as follows: when an organ is available for transplantation, a country-wide search is conducted for emergency patients. If such a patient is found within the cold ischemia time bounds, the organ is sent directly to that patient's hospital via road or air transportation without considering the RCC assignment hierarchies. If there is no suitable emergency patient, hierarchical matching begins with the waiting list of the hospital that introduces the donated organ to the system. If a suitable candidate is not found, the search is then expanded to other hospitals in the city. If there is no match on this level either, the search is expanded to the cities assigned to the donor's RCC. The last step is to search for a potential recipient 


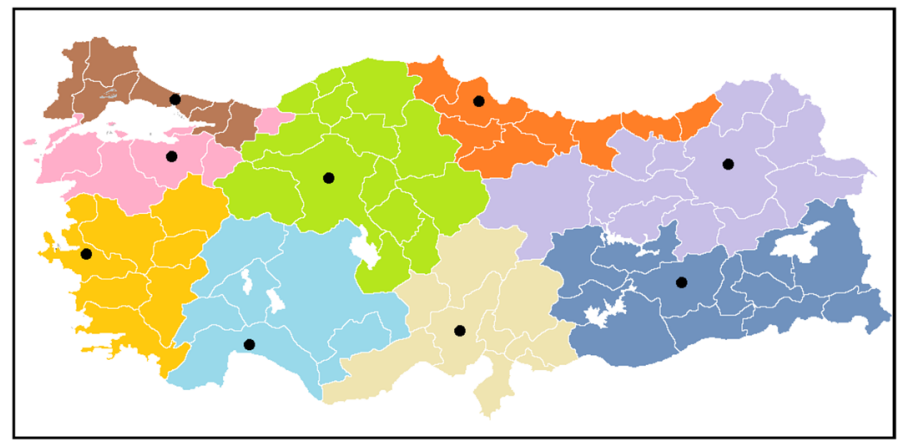

Fig. 1 Current RCC locations and assignments in Turkey

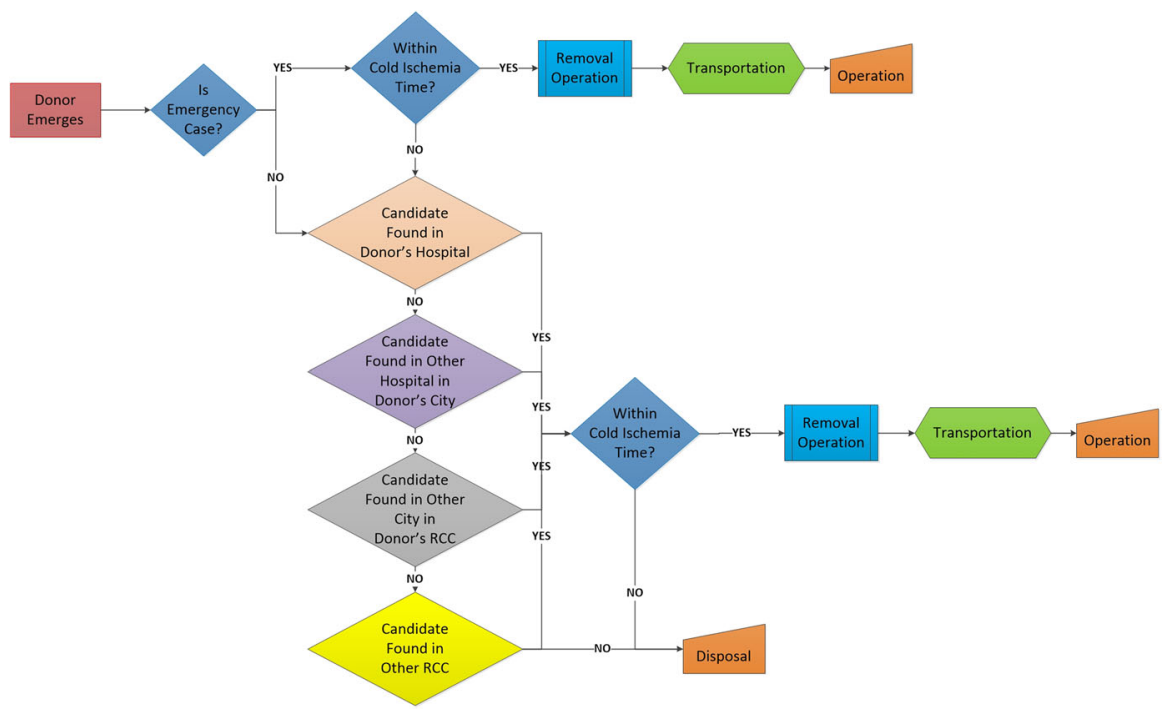

Fig. 2 Organ transplantation process in Turkey

across the country. The representation of Turkey's organ transplantation process can be found in Fig. 2.

The waiting lists of the RCCs and NCC are dynamically updated after each successful transplantation. For the RCC waiting lists, the city which received the organ is moved to the bottom of the list, so that it is ranked the last in the following organ matching. This allows the city that was ranked the second in the previous matching to move to the first position in the next organ matching within the same RCC. A similar approach is followed for the NCC waiting list too. The RCC that receives the donation is moved to the end of the list after the transplantation. This procedure is repeated continuously, and all waiting lists are updated dynamically at the end of each transplantation.

At the later steps of the hierarchical system, even if a suitable donor-recipient match is made, the travel time/distance may not be within the cold ischemia time 
bound, implying that a matched organ may not be transferred. While appropriate matching criteria greatly increase implantation success, if the organizational structure of the organ transplantation system is not well established, the overall system may not function so well. Therefore, logistics aspects of the system greatly affect the overall success.

A basic difference between an organ transplantation system like Turkey's and other hierarchical systems (like Italy's) is that in systems like Turkey's, RCCs are not responsible for transplantation; they are simply coordinators, contacting donor and recipient cities. This means that an organ does not have to travel to an RCC for transplantation; it travels to the patient's hospital directly. However, for managerial purposes, it is preferable that RCCs are located in the cities where there is at least one transplantation hospital.

\section{Related literature}

Healthcare problems have been studied in OR for almost 50 years (Brailsford 2005). Rais and Viana (2011) is one of the most recent review studies that examines the applications of OR methodologies in healthcare. According to this study, healthcare-related topics can be classified as emergency medical services, specialized healthcare services, blood banking, organ transplantation and pharmaceuticals. Location-allocation and set-covering models are widely used while solving these problems. In this section, first the studies that use these type of models are going to be discussed and then the organ transplantation literature is going to be reviewed in detail.

Blood banking is one of the most common issues studied in healthcare logistics. The supply chain design of the blood-banking network is addressed by location-allocation models by many authors (Nagurney and Masoumi (2012), Zahiri and Pishvaee (2017), Chaiwuttisak et al. (2016)). Sahin et al. (2007) developed a location-allocation model for blood services of the Turkish Red Crescent Society by decomposing the problem into subproblems and tackling the issue of locating service facilities and assigning demand points in three stages. Sha and Huang (2012) locate temporary blood collection facilities and assign donors to these locations in emergency situations while minimizing the overall system's costs. Jabbarzadeh et al. (2014) consider the problem of supplying blood to the ones in need after the occurrence of a disaster, and their study handles uncertainty by utilizing a scenario-based stochastic programming approach. Zahiri et al. (2014a) performed another study which incorporates uncertainty to the blood-banking problem and they adopt a robust stochastic programming approach. In the following year, Zahiri et al. (2015) focused on the blood distribution network design problem in which temporary and fixed facilities are located and donors are assigned to these points over a multi-period planning horizon while minimizing the overall cost.

There are many studies that focus on other healthcare topics within the locationallocation context. Araz et al. (2007) consider a multi-objective vehicle location model for emergency services such as ambulances or firefighting vehicles, using a coveringbased approach. The amount of population that can be covered by one vehicle and with backup are maximized, while the total travel distance exceeding the prespecified 
covering radius is minimized in this study. Syam and Cote $(2010,2012)$ both address specialized healthcare services by locating traumatic brain injury treatment and rehabilitation centers and allocating patients to them. Both studies minimize the overall cost by considering fixed costs associated with opening trauma centers, overhead costs of equipments and labor costs. Shariff et al. (2012) work on a healthcare facility planning problem in Malaysia, which tries to solve the problems arisen by the limited capacity of the facilities by using a capacitated maximal covering location problem. Mousazadeh et al. (2015) developed a bi-objective model for a pharmaceutical supply chain design problem where they decide the locations of the manufacturing/distribution centers along with the material flows in the network. The overall costs and unmet demand amounts are minimized in this problem, and as there is uncertainty in the system, a probabilistic programming approach is used.

Even though the healthcare problems are addressed via OR tools for a long period of time, organ transplantation-related studies are relatively recent. Even so depending on the objective, problem classifications have been changing for these studies. Liberatore and Nydick (2008) propose that for an analytical hierarchy process, organ transplantation should be considered separately from the general "therapy/treatment" category of healthcare problems. Rais and Viana (2011) state that organ donation studies should be categorized under "specialized and preventive healthcare" studies.

Despite the fact that it has been recently considered, the number of organ transplantation studies in OR is increasing. Most of these articles are country-based case studies that address policy issues. For example, Genc (2008) outlines managerial problems in organ procurement during the transplantation processes in Turkey. For Switzerland, Uehlinger et al. (2010) explore the effects of a new law on cold ischemia time and organ transportation and compare it with the former law using statistical data. Deffains and Ythier (2010) aim to find the optimal design for the stages of the transplantation processes in Spain. Friedewald et al. (2013) propose an alternative kidney allocation system for the USA to increase life expectancy of recipients. In this study, they conclude that any change in the allocation system impacts the donated kidney and recipient matchings, which affects the equity and utility of the allocation system. Drekic et al. (2015) represent the transplantation processes in Canada as a queuing system in which the waiting lists change periodically depending on the health conditions of the potential recipients. In their study, they aim to infer the likely waiting times of the patients on these lists and use this information as new data in transplantation systems.

Many studies analyze and evaluate alternative policies for managing waiting lists for liver transplantation, such as Ratcliffe et al. (2001) for the UK and Thompson et al. (2004) for the USA. Simulation models are applied to the organ transplantation system, as in a national organ allocation model for kidney transplantation in the USA (Taranto et al. 2000). Different from other allocation studies, Harper et al. (2000) focus on multiple kidney allocation policies and simulate their effects. Su and Zenios (2005) present stochastic programming models to determine a kidney allocation policy. Shechter et al. (2005) study a liver allocation policy using a discrete-event simulation model to test the performance of policy changes. Alagoz et al. (2009) discuss the organ allocation and acceptance in a comprehensive manner. The authors explain the organ transplantation system in general and comment on studies on optimization models for a liver allocation system. Bertsimas et al. (2013) focus on the fairness of organ 
allocation policies with a data-driven method, which is also applicable for the USA allocation system. They also consider the medical efficiency of allocations and the flexibility in policy design. Dag et al. (2016) try to determine which patient and donor data to consider for better heart matchings and aim to estimate survival length of the recipient after the procedure. Similarly, Misiunas et al. (2016) intend to parse the available patient and donor data correctly so that successful pairings between two parts can be obtained more accurately.

When the studies in the organ transplantation literature are investigated, the ones focusing on location-allocation problems are also commonly observed. One of the earliest works is by Stahl et al. (2005), and the study focuses on the liver transplantation system in the USA. The aim is to determine the regions in a better way so that the total and minimum intra-regional transplantation rate are maximized together. The authors propose a basic set partitioning type formulation, where regions are created considering cold ischemia times by depth search method. Kong et al. (2010) take the same system into consideration and utilize the very similar set partitioning perspective as Stahl et al. (2005), while only maximizing the total intra-regional flow. Their methods of creating regions also differ, and they are based on mixed-integer programming with a branch and price algorithm. Demirci et al. (2012) combine the two previous studies and use the objective function of Stahl et al. (2005) with both efficiency and equity concerns and generate the possible regions via a branch and price algorithm as Kong et al. (2010) develop. All three of these studies first determine and then provide alternative regions as an input to their mathematical formulations; however, we develop mathematical models that find the optimal clusters by itself using location-allocation approach instead of set partitioning.

Bruni et al. (2006) consider Italy's organ transplantation logistics, aiming to attain regional equity by minimizing the maximum size of the waiting lists among all regions. They also aim to minimize the total traveled distance to transplantation hospitals while deciding the regions and hospital locations. Beliën et al. (2012) study the Belgian case, focusing on locating transplantation centers for each type of organ, as they consider multiple organ types. In their mathematical formulation, they minimize the total transportation time between the organ becomes available and transplantation is done. First, they work with deterministic travel times; however, they relax this assumption and perform the same analyses with stochastic parameters as well. Smith et al. (2013) also consider both efficiency and equity by minimizing the populationweighted travel distance and deviation from the desired travel distance. Different from the other studies, they have levels of transplantation centers; hence, their model incorporates a hierarchical structure. Zahiri et al. have two studies (2014b, 2014c) on multi-period location-allocation of organ transplantation centers under uncertainty, in which they show computational results for the case of Iran. While the former study considers single-objective (minimize total cost), the latter aims to minimize total cost and transplantation time simultaneously. Both studies have multiple organ types and utilize multiple transportation modes such as ground or air network. The common characteristic of all these studies is that they either minimize the total transplantation process time or the travel distance between the donor and the recipient. Besides, we incorporate an objective function that maximizes the potential-weighted intra-regional flow to obtain better clusters in the network. 
One of the most relevant studies to this one is by Gentry et al. (2015). In this study, the aim is to redistrict the liver allocation system of the USA in a way that the disparity in liver availability among districts is minimized. In order to achieve this, the authors develop a binary integer program which minimizes the number of misdirected organs from the most urgent candidates. Afterward, they evaluate the optimal districts by using a discrete-event simulation that is patient oriented. The key performance indicators of the simulation are the number of misdirected allocations, transportation times, standard deviation of the urgency scores across the districts and number of waiting list/total deaths. Even if this study follows a similar modeling approach with ours, it only considers a single organ type and does not evaluate multiple transportation modes separately, where this claim is also valid for the majority of the previous studies. We enhance our model by including two modes (ground and air) since several problems (plane arrangement issues, weather conditions etc.) might make the ground transportation the only viable option at certain times. It also has a different objective than ours and develops the simulation model considering patients as the main entities, whereas we are more concerned with the organ deterioration during the process. Hence, the entity of our simulation model is organs, not patients.

We would like to emphasize that, the main objective of this study is not only finding optimal clusters for a hierarchical organ transplantation system, but also to observe the performance of the suggested clusters with respect to the uncertain environment of organ transplantation with a more realistic representation of the system, using the discrete-event simulation approach. We believe that it is not sufficient to suggest optimal clusters to the system without a simulation-based performance analysis, and especially without including hierarchical matching, emergency-related instances or vehicle availabilities. With the help of this tool, we are able to analyze and evaluate the performance of the suggested locations and allocations and also model real-life characteristics by including waiting lists and their dynamics in the problem. Finally, another important contribution of this study is that it utilizes a real-life data set from Turkey while aiming to improve the logistical framework for organ transplantation for this country.

In order to be able to compare this study with the previous ones in the locationallocation literature of organ transplantation systems, a summary is included in Table 1.

\section{Proposed models}

In this section, we present two optimization models: a basic model and an extended version to find optimal clusters for the organ transplantation system. The initial model only focuses on ground transportation, while the latter takes also air transportation into account. In both of these models, the number of clusters is fixed a priori. The objective of both models is to maximize the potential-weighted intra-regional flow within the same cluster.

Since the aim of this study is to analyze the effects of clusterings on the potential matches, the main motivation of the mathematical models is to develop clusters that maximize the potential of matchings at the first layer. Thus, the mathematical models 


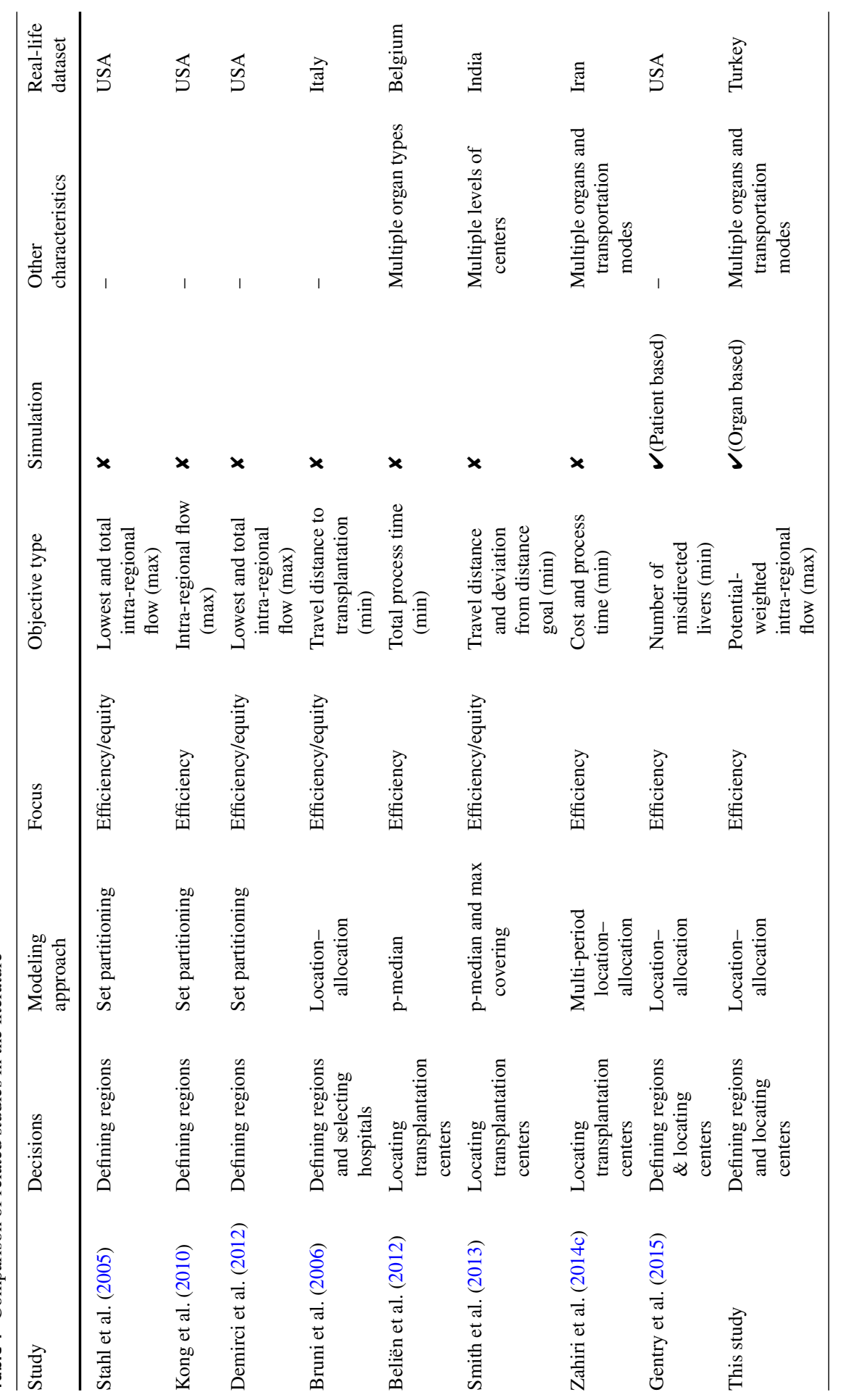


do not take the hierarchical matching process into consideration. This means that the mathematical formulation can only allocate the organs in an intra-regional manner. Since the supply is quite less than the demand, it is certain that the available organs are matched with a patient within the same region. In addition to that, the probabilistic events such as emergency cases, matching probabilities, vehicle availabilities are very hard to represent in such formulations. Because of these, the mathematical models cannot capture the hierarchical structure.

As a result of these points, the best way to represent the hierarchical matching process is through a simulation model. To achieve the desired outcomes, the outputs of the mathematical model (RCC locations, their allocated cities and helicopter assignments for the extended model option) are used as the inputs of the simulation model. By this way, the performance of the mathematical model solutions under uncertainty, with detailed system characteristics such as more than one donation from the same donor, a three-layered hierarchical structure, updated waiting lists for each organ and transplantation center and emergency case options can be observed. In the following sections, the details of both models are presented.

\subsection{Basic model: clustering for organ transplantation}

In this section, a basic model called Clustering for Organ Transplantation (COT) is introduced which is $\mathrm{O}\left(n^{3}\right)$ in the number of binary- and real-valued variables. This model is studied in order to account for any event that may lead to the inability to use helicopters or airplanes such as weather conditions and scheduling issues. Therefore, this model only considers the terrestrial distance between the cities in which the organ is introduced to the system and transplantation centers. For the managerial purposes, RCCs should be selected from cities that have at least one transplantation hospital. after:

Before presenting the model, we introduce the following notation to be used here-

Sets:

$M$ : set of the potential donor cities.

$N$ : set of cities with transplantation hospitals.

$R$ : set of potential RCC locations.

In real-life applications, $R \subseteq N \subseteq M$.

\section{Parameters:}

$O_{i}$ : total number of donated organs in city $i \in M$.

$D_{j}$ : total number of organ demand in city $j \in N$.

$b_{i j}$ : terrestrial travel time between cities $i \in M$ and $j \in N$.

$p$ : total number of RCCs to be located.

$T$ : maximum allowable travel time allowed to carry the organ

from the donor city to the transplantation center.

$a_{i j}=\left\{\begin{array}{l}1 \text { if city } i \in M \text { and } j \in M \text { share a border, } \\ 0 \text { otherwise. }\end{array}\right.$

\section{Decision variables:}

$z_{k}=\left\{\begin{array}{l}1 \text { if an RCC is located at } k \in R, \\ 0 \text { otherwise. }\end{array}\right.$ 
$x_{i k}=\left\{\begin{array}{l}1 \text { if city } i \in M \text { is assigned to the RCC in } k \in R \\ 0 \text { otherwise. }\end{array}\right.$

$y_{i j}^{k}=\left\{\begin{array}{l}1 \text { if cities } i \in M \text { and } j \in N \text { are both assigned to RCC } k \in R, \\ 0 \text { otherwise. }\end{array}\right.$

$m_{i j}^{k}=$ total number of potential organ matches between cities $i \in M$ and $j \in N$ in the region of RCC $k \in R$

$c_{i j}^{k}=$ auxiliary variable to control the contiguity; flow from city $i \in M$ to $j \in N$ in the region of RCC $k \in R$

The COT model can be written as follows:

$$
\begin{aligned}
& \max \sum_{i \in M} \sum_{j \in N} \sum_{k \in R}\left(1-\frac{b_{i j}}{T}\right) \cdot m_{i j}^{k} \\
& \text { s.t. } \sum_{k \in R} x_{i k}=1 \quad \forall i \in M \\
& \sum_{k \in R} z_{k}=p \\
& y_{i j}^{k}=x_{i k} \cdot x_{j k} \quad \forall i \in M, j \in N, k \in R \\
& x_{i k} \leq z_{k} \quad \forall i \in M, k \in R \\
& z_{k} \leq x_{k k} \quad \forall k \in R \\
& b_{i j} y_{i j}^{k} \leq T \quad \forall i \in M, j \in N, k \in R \\
& m_{i j}^{k} \leq O_{i} \cdot y_{i j}^{k} \quad \forall i \in M, j \in N, k \in R \\
& m_{i j}^{k} \leq d_{j} \cdot y_{i j}^{k} \quad \forall i \in M, j \in N, k \in R \\
& \sum_{j \in N} c_{i j}^{k} \cdot a_{i j}-\sum_{j \in N} c_{j i}^{k} \cdot a_{i j}=x_{i k} \quad \forall i \in M \backslash R, k \in R \\
& \sum_{j \in N} c_{j i}^{k} \cdot a_{i j} \leq(|M|-2) \cdot x_{i k} \quad \forall i \in M \backslash R, k \in R \\
& \sum_{j \in N} c_{j i}^{k} \cdot a_{i j} \leq(|M|-1) \cdot x_{i k} \quad \forall i \in R, k \in R \\
& x_{i k}, y_{i j}^{k}, z_{k} \in\{0,1\} \quad \forall i \in M, j \in N, k \in R \\
& m_{i j}^{k}, c_{i j}^{k} \geq 0 \quad \forall i \in M, j \in N, k \in R
\end{aligned}
$$

In the objective function (1), the potential-weighted intra-regional organ flow is aimed to be maximized, which will increase the possibility of a match in the earlier stages and hence decrease the amount of wasted organs. There are two major components of this objective function: one being the potential flow and the other being the weights of each match. First, as the organ supply is scarce compared to the demand amount, all donations are allocated to the recipients in the mathematical model. Therefore, maximizing the exact number of organ transplantations does not reveal the benefits of the new clustering approaches as the answer is a constant. Hence, instead the potential intra-regional organ matches are maximized in the objective func- 
tion, where a donation is accepted to be a potential match for each recipient city within the same region. As a result, the same supply amounts are counted multiple times in the objective value. Second, the quality of an organ in a match that happens in the same city with travel time 0 is not the same with another one that happens in $T$ time, since it deteriorates with time. Therefore, a weight is assigned to each match which is inversely proportional to the travel distance. The same city matches have weight 1 , whereas this decreases toward 0 as the travel time approaches to the upper limit, $T$.

Constraints (2) ensure that each city is assigned to exactly one RCC. The number of opened facilities is set to $p$ in constraint (3). Nonlinear set of constraints (4) define the possible donor-recipient matches between cities that are both assigned to the same RCC. Constraints (5) prevent assigning a city to a non-existing RCC. Constraints (6) ensure that if the city is an RCC, then it is assigned to itself. The maximum allowable time bound is satisfied via constraints (7). Constraints (8) and (9) define the organ flow between two cities by taking the minimum of organ supply and demand. The contiguity of the clusters is guaranteed by constraints (9)-(11). These set of constraints are taken from Shirabe (2009), which are constructed using a network flow-based approach. They make sure that the flow occurs between the cities that are adjacent to each other by utilizing the $a_{i j}$ parameter and the collection of cities that have a flow between is a continuous cluster. Finally, the last two constraints define the domain of the decision variables.

Since constraints (4) are nonlinear, they can be linearized by the following constraints (15) and (16), leading to the final mixed-integer linear programming formulation.

$$
\begin{array}{ll}
y_{i j}^{k} \leq \frac{x_{i k}+x_{j k}}{2} & \forall i \in M, j \in N, k \in R \\
y_{i j}^{k} \geq x_{i k}+x_{j k}-1 & \forall i \in M, j \in N, k \in R
\end{array}
$$

\subsection{Extended model: clustering for organ transplantation with helicopters}

In Clustering for Organ Transplantation with Helicopters (COT-H), air transport availability is integrated to the COT model. There are three additional parameters and two decision variables that are introduced to the updated model.

\section{Additional variables:}

$$
\begin{aligned}
& h c_{j}=\left\{\begin{array}{l}
1 \text { if a city } j \in N \text { has a helicopter, } \\
0 \text { otherwise. }
\end{array}\right. \\
& l_{i j}^{k}: \text { auxiliary variable that represents the multiplication of } m_{i j}^{k} \text { and } h c_{j} \\
& \text { Additional parameters: }
\end{aligned}
$$

\section{Additional parameters:}

$u_{i j}$ : air travel time between cities $i \in M$ and $j \in N$.

$h$ : total number of helicopters available.

$B N$ : a sufficiently big number.

Then, the mathematical model of COT-h becomes as follows:

$$
\max \sum_{i \in M} \sum_{j \in N} \sum_{k \in R}\left(1-\frac{b_{i j}}{T}\right) \cdot m_{i j}^{k}+\left(1-\frac{u_{i j}}{T}\right) \cdot l_{i j}^{k}-\left(1-\frac{b_{i j}}{T}\right) \cdot l_{i j}^{k}
$$




$$
\begin{aligned}
& \text { s.t. (2), (3), (5), (6), (8), (9), (10), (11), } \\
& \text { (12), (13), (14), (15), (16) } \\
& \sum_{j \in N} h c_{j}=h \\
& \left(b_{i j} y_{i j}^{k}\right)-\left(\left(b_{i j}-u_{i j}\right) h c_{j}\right) \leq T \quad \forall i \in M, j \in N, k \in R \\
& l_{i j}^{k}=m_{i j}^{k} \cdot h c_{j} \\
& \forall i \in M, j \in N, k \in R \\
& h c_{j} \in\{0,1\} \\
& \forall j \in N
\end{aligned}
$$

The objective function (17) slightly changes in this model even if it has the exact same idea behind it. While assigning the weights to the matches, the travel time of the specific transportation mode has to be selected. If the organ is transported via ground network, $l_{i j}$ gets the value 0 ; thus, the objective function considers the terrestrial travel times. On the other hand, when there is a organ transfer by a helicopter, first and third terms cancel each other out and the air travel times are taken into account while calculating the weights.

The additional constraint (18) fixes the total number of helicopters to $h$. Instead of constraint set (7) that bounds the travel time in a cluster, constraint set (19) is used. When a city does not have a helicopter, the formulation becomes the same as constraint set (7). On the other hand, when there exists a helicopter in the city, terrestrial travel times cancel each other out and air travel times determine the actual travel time. Nonlinear constraints (20) define the $l_{i j}^{k}$ variable and set it to the organ flow if there is a helicopter at city $j$ or to 0 if there is not. Finally, constraints (21) determine the binary variable restrictions.

As constraints (20) are nonlinear, they are required to be linearized. Instead of them, the following three constraints (22)-(24) can be used and the final version of the COT-H model can be obtained.

$$
\begin{array}{ll}
l_{i j}^{k} \leq h c_{j} \cdot B N & \forall i \in M, j \in N, k \in R \\
l_{i j}^{k} \leq m_{i j}^{k} & \forall i \in M, j \in N, k \in R \\
l_{i j}^{k} \geq m_{i j}^{k}-\left(\left(1-h c_{j}\right) \cdot B N\right) & \forall i \in M, j \in N, k \in R
\end{array}
$$

\subsection{Simulation model}

The aim of the simulation model is to observe the performance of the mathematical model by portraying the real-life characteristics of the problem such as hierarchical matchings, vehicle availabilities and emergency instances. The essential elements of this model are donors, types of donated organs, matching process and transporting the donated organ with alternative vehicles. This gives us the opportunity to translate the mathematical model's outputs into real- life situations, thus providing a better basis for the evaluation of its results. Since the simulation model consists of a complex and large model structure, a simplified version of it is provided in Fig. 3.

The process starts when a donor emerges. It is assumed that a donor can donate one, two or all of the following organs: kidneys, liver and heart. Then, the donor city 


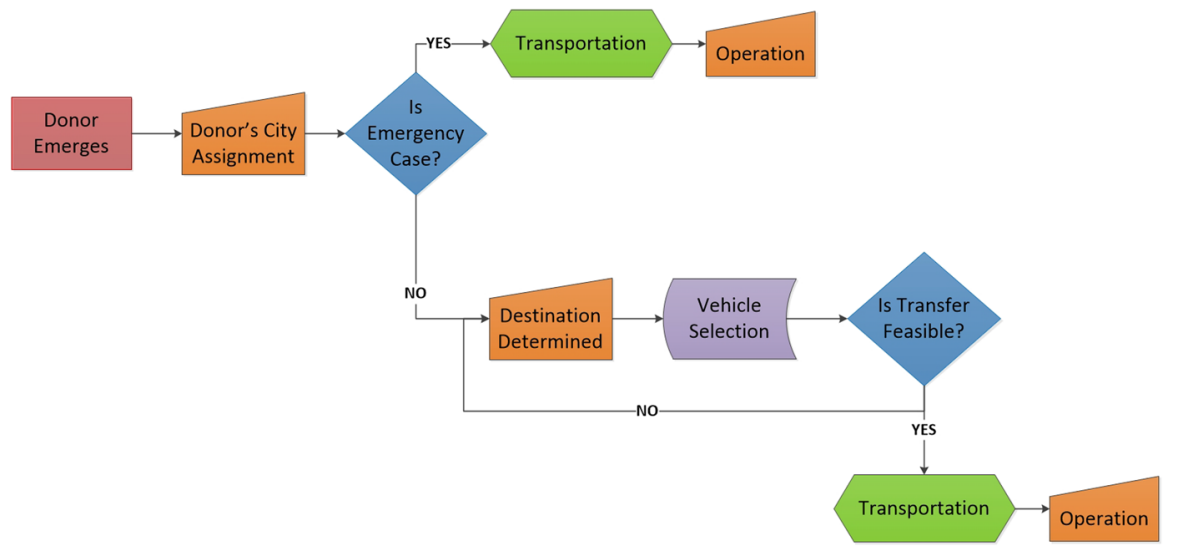

Fig. 3 High-level illustration of the simulation model

is assigned using an empirical distribution. If there is an emergency patient in the system, then the organ is sent to that recipient without consideration of the patient's location or rank in the waiting lists. This is usually done through air transportation to minimize time.

For non-emergency cases, the destination of the organ, i.e., the candidate recipient's city, is defined hierarchically. To do this, initially, the probability that there is a suitable recipient in the donor's city is determined. This is obtained via the probability $1-(1-$ $x / 100)^{N_{i}}$, where $x / 100$ is the probability that any particular donor is immunologically compatible with any particular recipient, and $N_{i}$ is the number of people waiting for a transplant in city $i$. In this case, the organ is directly sent to the recipient's hospital and transportation time is omitted. If a match cannot be found within the same city, the RCC of the donor's city begins to search for a candidate recipient from the list of hospitals that are assigned to it. In this step, the first-ranked city on the RCC waiting list (for the donated organ type) can be matched with the donor city using the same probability, i.e., $1-(1-x / 100)^{N_{i}}$. If the first-ranked city is not matched with the donor city, the search continues in the order of the cities in the waiting list. If the donor and recipient cities can be matched in the same RCC, then the selected city is moved to the end of the list and the transportation phase begins. If the corresponding RCC cannot find a match in its region, the NCC looks for a candidate recipient from the list of RCCs in a similar hierarchical manner.

After the organ is matched to a recipient, it can be transported via several options. If a helicopter is available in the donor city, then the organ is transported by air. Otherwise, if the distance between two cities is less than the organ's maximum allowable travel time, the organ is transferred by using ground network. Another option is that a commercial airplane can transport the organ if there is airline transfer between the two cities. Rarely, none of the transportation options can be used, which results in a search for a new recipient, using the same hierarchy.

Key performance indicators of the simulation model are determined to be the percentage of successful transplantations within the same RCC (first level of the hierarchy), organ travel times, number of disposed organs and number of trips done by 
cars, helicopters and airplanes, which will be discussed in the computational analysis section.

\section{Computational results}

In this section, we present the numerical results of the mathematical and simulation models using a real-life data set of Turkey, which is available at www.ie.bilkent.edu. tr/ bkara. First, we start with explaining the steps of data collection in the following section.

\subsection{Data}

There are 81 cities in Turkey, and as they all host potential donors, they are all considered as donor cities. The recipients are the set of cities where transplantation operations can be conducted. As the number of transplantation centers is limited, there are only 20 candidates for recipient cities. The details of these centers are presented in Table 2.

The data for ground travel time between two cities are taken from Turkey's General Directorate of Highways. Since Turkey is not a geographically large country when flight times are considered, we accepted the travel time between Ankara and Istanbul as an basis while determining helicopter flight duration between two cities. The terrestrial travel time between Ankara and Istanbul is approximately $4.5 \mathrm{~h}$ and helicopter travel time is $1.5 \mathrm{~h}$; therefore, flight times between two cities are assumed to be one-third of the terrestrial travel times for the remaining pairs.

The cold ischemia time of heart, liver and kidney is 5,12 and $18 \mathrm{~h}$, respectively. According to the expertise and experience, the organ removal and implantation surgery times are determined as detailed in Table 3. When these durations are subtracted from the ischemia times of corresponding organs, approximate transportation time for a heart is found to be $220 \mathrm{~min}$, whereas it is $405 \mathrm{~min}$ for a liver and $570 \mathrm{~min}$ for a kidney (Dr. Kahveci, 2012).

In the mathematical model, number of donated organs at each city and number of recipients on the waiting lists of each transplantation center are required to be able to determine the organ flow. We estimated the number of potential organ donations from cadavers for each city by utilizing the data retrieved from governmental database (Republic of Turkey Ministry of Health 2012) and using the ratio of donations from cadavers in 2011 per 10,000 people for each RCC. We obtained the total donor ratio for each RCC by using the information on the total number of organ donations from cadavers and living donors in 2011.

To estimate the total number of recipients for each transplantation center, we assumed that the number of patients in each organ transplantation hospital is similar and we scaled the data for the total number of successful organ transplantation ratios for each RCC to 19,403 patients (the number waiting for a transplant in 2011). Then, we scaled the total number of hospitals that can perform organ transplantation in that city to the number of patients in the respective RCC. This gave us the approximate number of patients on a waiting list per city. 
Table 2 Transplantation centers for organ types

\begin{tabular}{|c|c|}
\hline City & Number of centers \\
\hline \multicolumn{2}{|c|}{ Kidney transplantation centers } \\
\hline Adana & 2 \\
\hline Ankara & 10 \\
\hline Antalya & 2 \\
\hline Bursa & 1 \\
\hline Denizli & 1 \\
\hline Edirne & 1 \\
\hline Erzurum & 1 \\
\hline Eskişehir & 1 \\
\hline Gaziantep & 2 \\
\hline Istanbul & 21 \\
\hline Izmir & 7 \\
\hline Kahramanmaraş & 1 \\
\hline Kayseri & 1 \\
\hline Kocaeli & 2 \\
\hline Konya & 2 \\
\hline Malatya & 1 \\
\hline Mersin & 1 \\
\hline Samsun & 1 \\
\hline Trabzon & 1 \\
\hline
\end{tabular}

Liver transplantation centers

\begin{tabular}{ll}
\hline Adana & 5 \\
Ankara & 10 \\
Antalya & 3 \\
Bursa & 1 \\
Diyarbakır & 1 \\
Erzurum & 1 \\
Istanbul & 9 \\
Izmir & 3 \\
Samsun & 1 \\
\hline Heart transplantation centers & \\
\hline Adana & 1 \\
Ankara & 6 \\
Antalya & 1 \\
Istanbul & 4
\end{tabular}

In the simulation model, the inter-arrival time of the donors is defined by exponential distribution with the mean of 2.01, considering the total number of donors in the previous year. Since the system does not track the number of patients and donors, 
Table 3 Cold ischemia time for heart, liver and kidneys

\begin{tabular}{lllll}
\hline Organ & $\begin{array}{l}\text { Ischemia } \\
\text { time }(\mathrm{h})\end{array}$ & $\begin{array}{l}\text { Organ removal } \\
\text { surgery time }(\mathrm{min})\end{array}$ & $\begin{array}{l}\text { Organ implantation } \\
\text { surgery time }(\mathrm{min})\end{array}$ & $\begin{array}{l}\text { Average transportation } \\
\text { time }(\mathrm{min})\end{array}$ \\
\hline Heart & 5 & 10 & 70 & 220 \\
Liver & 12 & 45 & 270 & 405 \\
Kidney & 18 & 60 & 450 & 570 \\
\hline
\end{tabular}

Distribution of Donated Organs

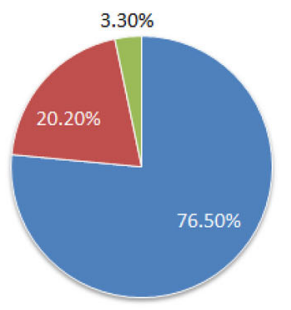

Distribution of Emergent Cases

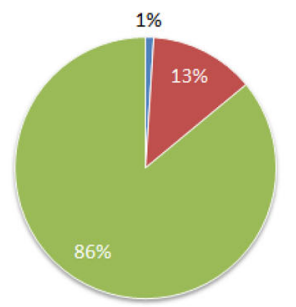

Kidney

- Liver

meart

Fig. 4 Percentages of donated organs and emergent cases in the system

we must omit seasonality effects on these values. A donor city is assigned to the arriving organ with an empirical distribution, by setting the probability to be the ratio of potential number of donors in that city to the total donation amount. The percentage of each type of donated organ in standard and emergency cases in 2011 are given in Fig. 4. We assume that all cities have the same matching probability of $15 \%$, a number that is obtained by determining the average of Turkey's match ratio, that is between $10 \%$ and 20\% (Aksiyon 2012). The travel times of the planes that are utilized in the simulation model are calculated by considering the average flight times between the cities. If a direct flight is not available between a pair of cities, then the related waiting times at the transfer airport are also considered in the data preparation process.

\subsection{Results of mathematical models}

The mathematical models were solved by using IBM ILOG Cplex Optimization Studio v12.5. The results consist of three main parts. In the first section, the minimum number of required RCCs is determined for each organ. Afterwards, the number of facilities is fixed to these numbers and the minimum travel times are calculated. In the last section, air transportation is introduced to the mathematical model and the minimum number of helicopters required for each organ is found. Each of these analyses aims to maximize the potential-weighted matching amounts at the regional level. The dashes in the following tables denote the settings those components are not considered.

In the model, maximum allowable travel time is determined between each transplantation center and all the cities in a cluster. The model arbitrarily selects one of the cities with a transplantation center as the RCC. Thus, within each cluster, RCC locations can be changed without affecting optimality. Not all the cities are assigned to their nearest RCCs, which arises mainly because the potential-weighted intra-cluster 
Table 4 Minimum Number of RCC's Required

\begin{tabular}{lllll}
\hline & $\begin{array}{l}\text { Current } \\
\text { Case 1 }\end{array}$ & Instance 1 & Instance 2 & Instance 3 \\
\hline Organ type & - & Heart & Liver & Kidney \\
Travel time bound $(T)$ & 481 & 220 & 405 & 570 \\
Fixed number of RCC's $(p)$ & 9 & - & - & - \\
Minimum number of RCC's $(p)$ & - & Infeasible & 6 & 4 \\
Objective value & $90,427.27$ & Infeasible & $116,153.21$ & $207,758.84$ \\
\hline
\end{tabular}

flows are maximized. In the COT-H model, the helicopters can be assigned to candidate RCC locations as the medical team responsible for harvesting the donated organs are only available at these points.

Since the maximum allowable travel time differs for each organ type, optimization model is run for $T=220 \mathrm{~min}$ (heart), $T=405 \mathrm{~min}$ (liver) and $T=570 \mathrm{~min}$ (kidney) to find the minimum number of required RCCs. First the current system is evaluated with the mathematical model, whose RCC locations and allocations can be found in Fig. 1. Currently, there are 9 RCCs and the minimum of the maximum time bound between each donor and recipient city is $481 \mathrm{~min}$. Thus, the current clusters cannot satisfy heart's and liver's maximum allowable travel time bounds by only road transportation. It should also be noted here that some of the clusters in the current system are not contiguous; hence, the related constraints are not included in the analysis of this setting.

As seen in Table 4, Turkey cannot be clustered in a way that the maximum allowable travel time of heart is met in every matching pair; therefore, the respective model turns out to be infeasible. On the other hand, the minimum number of RCCs required for liver and kidney is 6 and 4, respectively. After obtaining these data, the number of RCCs is fixed to the corresponding $p$ values, namely 6 for $T=405$ (Instance 2 ) and 4 for $T=570$ (Instance 3 ), and the original mathematical model is run to maximize potential-weighted intra-regional organ flow in the system. Outcomes of these instances can be found in Table 4.

Next, the travel time bounds are minimized while keeping the number of clusters fixed at the values that are obtained in Table 4. Since there is no feasible solution for $T=220$ (heart), the minimum possible $T$ value is determined without any restrictions on the number of RCCs. It is found out that the minimum of longest allowable travel time is 314 min which can be achieved with at least 8 RCCs. The illustration of this instance can be seen in Fig. 5. This analysis is conducted to guide the decision makers to open new heart transplantation centers in the future as this organ cannot be transported in the whole country with ground network.

For the remaining two cases, when the number of RCCs is fixed at 6 and 4, it is observed that the travel time bounds can be decreased to 374 and 537 min from 405 and $570 \mathrm{~min}$, respectively. The objective values of these instances are quite similar to the original time bounds of liver and kidney, which indicates that the travel times can be decreased with the same number of RCCs while maintaining the weighted intra-regional flow at the same level. These results can be seen in Table 5. 


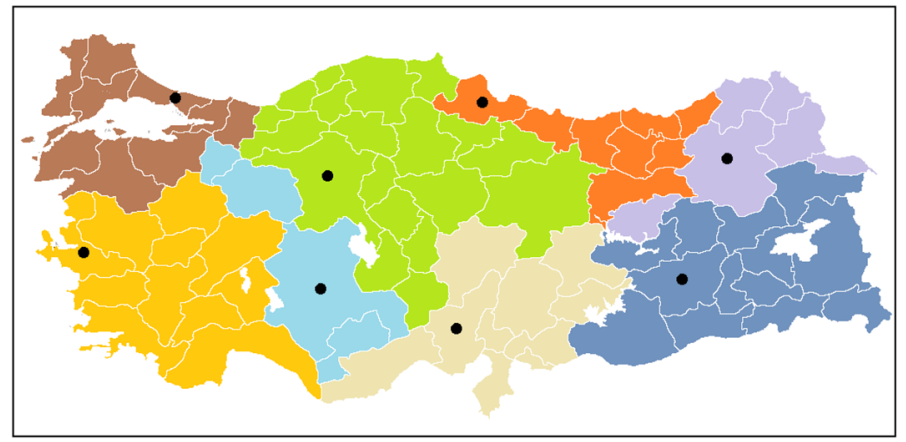

Fig. 5 COT for $p=8$ and allowable transportation time $T=314 \mathrm{~min}$

Table 5 Minimum travel times

\begin{tabular}{lllll}
\hline & $\begin{array}{l}\text { Current } \\
\text { case 1 }\end{array}$ & Instance 4 & Instance 5 & Instance 6 \\
\hline Fixed number of RCC's $(p)$ & 9 & 8 & 6 & 4 \\
Minimum travel time $(T)$ & 481 & 314 & 374 & 537 \\
Objective Value & $90,427.27$ & $87,570.55$ & $104,086.59$ & $191,793.25$ \\
\hline
\end{tabular}

In the next step, as heart's travel time bound cannot be met, air transportation is introduced to the original model. Two different approaches are utilized in this section. There are 5 cities in Turkey (Adana, Ankara, Antalya, İstanbul, İzmir) with heart transplantation centers; however, none of these are located in the eastern region of the country. Since this region is quite large, $T=220 \mathrm{~min}$ bound cannot be satisfied due to the lack of facilities in this area. This issue can be overcame by selecting one RCC from a candidate set of eastern cities (Diyarbakir, Erzurum, Gaziantep and Malatya) that maximizes the objective function alongside to the aforementioned 5 RCCs with heart transplantation centers. Alternatively, these 5 RCCs can again be fixed and 3 more locations can be selected from the remaining 15 candidate RCC locations.

Initially, minimum number of required helicopters for these approaches are found as 11 and 5, respectively. On the other hand, when the current structure is analyzed, it is observed that $T=220$ (heart) min bound can be met with at least 14 helicopters. This indicates that a more structured clustering approach can use less RCCs and helicopters to satisfy heart travel time and decrease the operational costs, even though monetary aspect of the network is not investigated in this study.

Since the matchings can be performed for liver and kidney cases in the proposed cases via ground network, helicopters are actually not required. In order to perform analyses on these settings, the number of RCCs is decreased by one, and this resulted in the necessity of a single helicopter for the liver and 3 extra helicopters for the kidney case. Next, these numbers are fixed and the original model is run to obtain the maximum potential-weighted intra-regional organ flow. A comparison of the objective values of current and proposed systems reveals that better clusters that have higher objective values can be obtained with less RCCs. The results of these models can be found in Table 6 . 
Table 6 Minimum number of helicopters

\begin{tabular}{llllll}
\hline & $\begin{array}{l}\text { Current } \\
\text { case 2 }\end{array}$ & Instance 7 & Instance 8 & Instance 9 & Instance 10 \\
\hline Organ type & Heart & Heart & Heart & Liver & Kidney \\
$\begin{array}{l}\text { Travel time bound }(T) \\
\text { Fixed number of }\end{array}$ & 220 & 220 & 220 & 405 & 570 \\
$\begin{array}{l}\text { RCC's }(p) \\
\begin{array}{l}\text { Minimum number of } \\
\text { helicopters (h) }\end{array}\end{array}$ & 14 & 6 & 8 & 5 & 3 \\
\begin{tabular}{l} 
Objective Value \\
\hline
\end{tabular} & $5,826.02$ & $7,484.24$ & $6,362.54$ & $7,886.79$ & $12,399.88$ \\
\hline
\end{tabular}

Table 7 Number of clusters satisfied in different $T$ values, road transportation

\begin{tabular}{lllll}
\hline Cluster structure & Number of clusters & \multicolumn{4}{l}{ Number of clusters satisfied within $T$} \\
\cline { 3 - 5 } & & Instance 4 & Instance 2 & Instance 3 \\
\hline Instance 4 $(T=314)$ & 8 & - & 8 & 8 \\
Instance 2 $(T=405)$ & 6 & 1 & - & 6 \\
Instance 3 $(T=570)$ & 4 & 0 & 1 & - \\
\hline
\end{tabular}

As a single location-allocation structure can be implemented through the network, performance of the each cluster for different organ types is also analyzed. In the cases where only ground transportation is allowed, it is indicated that no clustering structure can satisfy the heart transportation time bound $(T=220 \mathrm{~min})$. The closest time bound $T=314$ min obviously satisfies the liver and kidney bounds too. When the clusters within liver time bound $(T=405 \mathrm{~min})$ are analyzed, it is observed that out of 6 clusters, one of them satisfies the $T=314 \mathrm{~min}$ bound. For the 4 clusters within kidney time bound ( $T=570 \mathrm{~min}$ ), one of them satisfies $T=405 \mathrm{~min}$ time bound, whereas none is within $T=314$ min bound.

In the cases where both road and air transportation are allowed, clearly both clusterings for $T=220$ min satisfy liver and kidney time bounds. In the liver setting, one of the 5 clusters can meet heart time bound. Out of 3 clusters formed for kidney time bound, none is within heart and only one is within liver time bounds. The summary of these analyses can be found in Tables 7 and 8, and decision makers can evaluate these results in order to select the best clustering for the organ transplantation logistics network of the entire country.

After analyzing the mathematical model results, we now compare the current system and proposed solutions utilizing the simulation model.

\subsection{Results of simulation model}

We used Arena Simulation Software 13.5 to model the processes described in Sect. 4.3. In the simulations, run length is set to 365 days and replication length is fitted to 50 to achieve a sufficiently large sample size for valid statistics which stay within the $95 \%$ 
Table 8 Number of clusters satisfied in different $T$ values, road and air transportation

\begin{tabular}{llllll}
\hline Cluster structure & \multirow{2}{*}{$\begin{array}{l}\text { Number of } \\
\text { clusters }\end{array}$} & \multicolumn{4}{l}{ Number of clusters satisfied within $T$} \\
\cline { 3 - 6 } \cline { 3 - 5 } & & Instance 7 & Instance 8 & Instance 9 & Instance 10 \\
\hline Instance $7(T=220)$ & 6 & - & 6 & 6 & 6 \\
Instance $8(T=220)$ & 8 & 8 & - & 8 & 8 \\
Instance $9(T=405)$ & 5 & 1 & 1 & - & 5 \\
Instance $10(T=570)$ & 3 & 0 & 0 & 1 & - \\
\hline
\end{tabular}

Table 9 Simulation model results without helicopters

\begin{tabular}{lllll}
\hline & $\begin{array}{l}\text { Current } \\
\text { case 1 }\end{array}$ & Instance 4 & Instance 2 & Instance 3 \\
\hline Organ Type & - & - & Liver & Kidney \\
Number of RCC's $(p)$ & 9 & 8 & 6 & 4 \\
Travel time bound $(T)$ & 481 & 314 & 405 & 570 \\
Average organ travel time & 89.9 & 95.43 & 105.72 & 135.24 \\
Half width & 0.45 & 0.68 & 0.51 & 0.76 \\
Average total organ output & 4,082 & $4,081.92$ & $4,082.08$ & $4,083.62$ \\
Half width & 20.54 & 20.27 & 19.78 & 20.18 \\
Average matching in first layer & 3,648 & $3,811.42$ & $3,921.20$ & $3,979.26$ \\
Half width & 19.91 & 19.65 & 18.98 & 19.68 \\
Percentage of matching in first layer $(\%)$ & 89.37 & 93.38 & 96.95 & 97.45 \\
Average number of disposed organ & 1.80 & 0.26 & 0.22 & 0.00 \\
Half width & 0.24 & 0.14 & 0.13 & 0.00 \\
Average number of plane trips & 99 & 68.64 & 48.06 & 61.24 \\
Half width & 3.39 & 2.44 & 2.05 & 2.25 \\
\hline
\end{tabular}

confidence interval. The average values of the performance measures with their half widths are given in the following tables.

In Table 9, the outcomes of the simulation model that only considers ground transportation are given. The current system with $T=481$ min and 9 RCCs results in $89.37 \%$ matching rate at the first level of the hierarchy. Even if the average total organ output is approximately the same for all models, this match percentage increases to $93.38 \%$ when there are 8 RCCs with $T=314$ min bound. For the other two proposed clusters, matching at the first level further increases to $96.95 \%$ for liver and $97.45 \%$ for kidney settings. Another improvement observed in the proposed systems is the decrease in the average number of plane trips, which indicates that the new clusters are not as much dependent to the commercial flights as it is in the current system. This is an indirect benefit that will be gained since these flights are not in control of the organ transplantation system and fewer number of plane trips is desirable. From the results, it can also be concluded that when there are less RCCs, average organ travel time increases due to larger regional boundaries. 
In Table 10, the results of the simulation models of current and proposed systems are compared while considering both air and ground transportation options. The current system with 9 RCCs and 14 helicopters provides $89.07 \%$ matching rate within the same RCCs for heart; however, this percentage can be increased to $96.27 \%$ with 6 RCCs and 11 helicopters and $93.46 \%$ with 8 RCCs and 5 helicopters. When the three settings for this organ are compared, it can be seen that both of the proposed solutions require fewer helicopters. It is observed that even if the number of helicopters is decreased by only 3 in the first system, there are $45 \%$ less trips on average. This indicates that the ground network of the proposed systems is established better and the current network greatly depends on helicopter transportation. On the other hand, dependence on the air network decreases in the proposed systems, which is a desired outcome, since helicopters may be unavailable at times due to various reasons. Another observation made is that the average number of disposed organs decreases in the proposed networks compared to the current one. Finally, as it is expected, the average organ travel time increases slightly when the air transportation option is used less frequently in the proposed systems.

For the liver case, when the number of RCCs is decreased from 6 to 5 and 1 helicopter is introduced to the system, it is observed that the intra-regional matching percentage decreases from $96.95 \%$ to $91.45 \%$. Similarly, for the kidney case when there exists one less RCC in the system and 2 extra helicopters, this rate decreases from 97.45 to $91.09 \%$. In both situations, even number of helicopter trips are increased compared to the heart cases, the average organ travel times are higher since there are less RCCs and the clusters have larger boundaries. The details of the results of all systems can be found in Table 10.

\section{Model variations to account for different organ types}

Up until now, different clusters with varying number of RCCs are proposed for each organ type. However, it can be said that locating different RCCs in a single network depending on the organ type is practically not that much applicable. Instead of this approach, it could be aimed to develop a mathematical model which considers maximizing the potential flow of all organ types simultaneously.

In the COT model, intra-regional flow is handled as a whole and independent from the organ type. As a result, it is observed that kidney time bound can be satisfied with 4 RCCs mentioned as in the previous section. However, there a few clusters, the regions are quite wider than usual and this decreases the applicability and effectiveness of the network in terms of liver and heart flows. On the other hand, when the current case is analyzed, it is found out that even with 9 RCCs, liver and heart time bounds are not satisfied in some regions of the country. Therefore, it is required to come up with a network that has enough number of clusters with acceptable widths and satisfies all organs' travel time bounds as much as possible.

At this point, the minimum maximum allowable travel time bound for road transportation, that is $314 \mathrm{~min}$, is taken into consideration, and it is known that the best clustering can be obtained with 8 RCCs for this bound. Therefore, it is decided to locate 8 RCCs in this network. The aim of this extension is to make sure each possible 
Table 10 Simulation model results with helicopters

\begin{tabular}{|c|c|c|c|c|c|}
\hline & $\begin{array}{l}\text { Current } \\
\text { case } 2\end{array}$ & Instance 7 & Instance 8 & Instance 9 & Instance 10 \\
\hline Organ type & Heart & Heart & Heart & Liver & Kidney \\
\hline Number of RCC's (p) & 9 & 6 & 8 & 5 & 3 \\
\hline $\begin{array}{l}\text { Number of available } \\
\text { helicopters }(h)\end{array}$ & 14 & 11 & 5 & 1 & 3 \\
\hline Travel time bound $(T)$ & 220 & 220 & 220 & 405 & 570 \\
\hline Average organ travel time & 78.53 & 97.58 & 107.92 & 129.88 & 144.16 \\
\hline Half width & 0.5 & 0.74 & 0.70 & 0.78 & 0.81 \\
\hline $\begin{array}{l}\text { Average total organ } \\
\text { output }\end{array}$ & 4091 & 4081.68 & 4083.26 & 4090.12 & 4097.78 \\
\hline Half width & 19.53 & 21.22 & 20.27 & 18.94 & 19.13 \\
\hline $\begin{array}{l}\text { Average matching in first } \\
\text { layer }\end{array}$ & 3644 & 3929.34 & 3816.16 & 3740.50 & 3732.60 \\
\hline Half width & 17.91 & 20.46 & 18.77 & 18.77 & 17.31 \\
\hline $\begin{array}{l}\text { Percentage of matching in } \\
\text { first layer }(\%)\end{array}$ & 89.07 & 96.27 & 93.46 & 91.45 & 91.09 \\
\hline $\begin{array}{c}\text { Average number of } \\
\text { disposed organ }\end{array}$ & 12.84 & 6.76 & 8.84 & 0.02 & 0.02 \\
\hline Half width & 0.78 & 0.78 & 0.95 & 0.04 & 0.04 \\
\hline $\begin{array}{l}\text { Average number of } \\
\text { helicopter trips }\end{array}$ & 415 & 226.38 & 26.34 & 7.36 & 92.12 \\
\hline Half width & 6.7 & 5.01 & 1.96 & 0.78 & 3.38 \\
\hline $\begin{array}{l}\text { Average number of plane } \\
\text { trips }\end{array}$ & 52 & 49.40 & 80.84 & 147.16 & 126.04 \\
\hline Half width & 2.34 & 1.87 & 2.41 & 3.68 & 3.26 \\
\hline
\end{tabular}

matching satisfies the kidney travel time bound $(T=570 \mathrm{~min})$ and increase the flow of all organ types simultaneously.

\subsection{Mathematical model}

In this subsection, the extended model that is called Clustering for Organ Transplantation Extension (COT-E) is introduced. In addition to the sets, parameters and decision variables of the COT model, some new items are introduced to the system.

\section{Additional set:}

$S$ : set of the organs (1=heart, $2=$ liver, $3=$ kidney)

\section{Additional parameters:}

$\alpha$ : maximum percentage of supply that can be allocated to an RCC.

$\beta$ : minimum percentage of supply that can be allocated to an RCC.

$T_{s}$ : maximum allowable travel time of organ $s \in S$.

$f_{i j}^{s}$ : amount of flow between cities $i \in M$ and $j \in N$ for organ $s \in S$ (which can be defined as minimum of supply and demand of cities). 


\section{Additional variable:}

$w_{i j}^{k s}=\left\{\begin{array}{l}1 \text { if cities } i \in M \text { and } j \in N \text { are both assigned to RCC } k \in R \text { and within } \\ \text { the travel time of organ } s \in S, \\ 0 \text { otherwise. }\end{array}\right.$

The mathematical formulation can be written as follows:

$$
\begin{array}{ll}
\max & \sum_{i \in M} \sum_{j \in N} \sum_{k \in R} \sum_{s \in S}\left(1-\frac{b_{i j}}{T_{s}}\right) \cdot f_{i j}^{s} \cdot w_{i j}^{k s} \\
& \quad(2),(3),(5),(6),(7),(10),(12),(13) \\
b_{i j} y_{i j}^{k} \leq T_{s^{\prime}}-\left(T_{s^{\prime}}-T_{s}\right) \cdot w_{i j}^{k s} \quad & \forall i \in M, j \in N, k \in R, s \in S, s^{\prime} \in S: s^{\prime}>s \\
w_{i j}^{k s} \leq y_{i j}^{k} & \forall i \in M, j \in N, k \in R, s \in S \\
\sum_{i \in M} O_{i} \cdot x_{i k} \leq \alpha \cdot \sum_{i \in M} O_{i} & \forall k \in R \\
\sum_{i \in M} O_{i} \cdot x_{i k} \geq \beta \cdot \sum_{i \in M} O_{i} \cdot z_{k} & \forall k \in R \\
w_{i j}^{k s} \in\{0,1\} & \forall i \in M, j \in N, k \in R, s \in S
\end{array}
$$

In the objective function (25), the potential-weighted flow between each alternative pair is maximized. This time, the weight is determined specifically for each organ by using the $T_{s}$ parameter. In order to determine which matchings satisfy which organ's time bound, constraints (26) are developed. Here, if a matching in an RCC satisfies organ $s$ 's time bound, then the $T_{s^{\prime}}$ values, which are the time bounds of organs that have larger $T$ values, cancel each other. Otherwise, the second term of the right-hand side drops, and all of the matchings are forced to satisfy the largest time bound. In constraints (27), the decision variable $w$ is forced to 0 if two cities are not assigned to the same RCC.

In the preliminary testings of this model, it is observed that the number of cities in a cluster can be either too much or too few. To be able to obtain more balanced solutions, the supply amount allocated to each cluster is limited with an upper bound $\alpha$ and a lower bound $\beta$ percentage via constraints (28) and (29). This approach is more suitable than limiting the number of cities in a cluster because organ donation amounts hugely vary between the cities and they cannot be treated equally. Finally, the domain of the new variable is defined in (30).

\subsection{Computational results}

The sets of donor cities, recipient cities and alternative RCC locations are determined as they were in the COT model. Similarly, the supply and demand amounts and the terrestrial travel times take the same values as before. $T_{S}$ values are also defined in the same way, but instead of changing the $T$ value for each run, an organ index is added to the parameter. The indexes 1,2, 3 represent heart, liver and kidney; therefore, $T_{1}$ is 
Table 11 Potential organ flows obtained with COT and COT-E Models

\begin{tabular}{|c|c|c|c|c|c|c|c|}
\hline & & \multicolumn{2}{|l|}{ Heart } & \multicolumn{2}{|l|}{ Liver } & \multicolumn{2}{|l|}{ Kidney } \\
\hline & & COT & COT-E & COT & COT-E & COT & COT-E \\
\hline \multirow[t]{3}{*}{$\alpha=45 \%$} & $\beta=1 \%$ & 147,797 & 156,215 & 275,526 & 285,486 & 298,766 & 290,742 \\
\hline & $\beta=2 \%$ & 151,940 & 154,549 & 247,503 & 251,765 & 288,539 & 283,956 \\
\hline & $\beta=3 \%$ & 127,209 & 140,779 & 228,742 & 237,010 & 258,914 & 253,608 \\
\hline \multirow[t]{3}{*}{$\alpha=40 \%$} & $\beta=1 \%$ & 143,658 & 150,368 & 259,575 & 262,407 & 285,953 & 279,643 \\
\hline & $\beta=2 \%$ & 138,907 & 149,999 & 248,386 & 256,308 & 283,368 & 276,800 \\
\hline & $\beta=3 \%$ & 132,448 & 141,290 & 232,654 & 241,894 & 252,388 & 246,484 \\
\hline \multirow[t]{3}{*}{$\alpha=35 \%$} & $\beta=1 \%$ & 144,219 & 147,343 & 230,707 & 238,834 & 248,549 & 247,949 \\
\hline & $\beta=2 \%$ & 122,321 & 129,668 & 202,568 & 214,622 & 230,378 & 224,493 \\
\hline & $\beta=3 \%$ & 127,539 & 131,586 & 216,016 & 218,378 & 238,174 & 229,749 \\
\hline
\end{tabular}

set to $220, T_{2}$ to 405 and $T_{3}$ to $570 \mathrm{~min}$. As it is mentioned before, parameter $p$ is set to 8 .

While determining the $\alpha$ and $\beta$ values, an analysis is developed. It is observed that without any limitations, generally the COT-E model forms one cluster which contains approximately $70 \%$ of the total supply, while some of the others are left with 1 or 2 small cities which generate the $0.1 \%$ of the overall supply amount. The initial $\alpha$ value is accepted as $45 \%$, and it is decreased to $40 \%$ and $35 \%$, systematically. The 3 largest cities in Turkey: Istanbul, Izmir and Ankara, contain the $31 \%$ of the overall supply. Hence, it is decided that decreasing $\alpha$ value further would result in excessive limitation on the problem and also might cause infeasibilities. Similarly for the $\beta$ parameter, initial value is taken as $1 \%$ to get rid of the solutions with clusters containing $0.1 \%$ of the total supply. Then, this value is increased to $2 \%$ and $3 \%$, methodically. Larger $\beta$ values are not investigated furthermore in order not to highly limit the solution pool.

In the computational studies stage, first the original COT model is run with the explained $\alpha$ and $\beta$ bounds to determine the base cases for each iteration. In this model, potential-weighted intra-regional kidney flow is maximized, and according to the network, all three organ types' flow amounts are recorded. Then, COT-E model is run with the same $\alpha$ and $\beta$ combinations and the potential-weighted flow of each organ type is maximized in the objective function. The results of both models are compared in Tables 11 and 12. In Table 11, the potential flow amounts for each organ type are given for COT and COT-E models, whereas in Table 12, the changes in potential flows that are obtained with COT-E model are shown in percentages.

All of the instances are run with a time limit of $3 \mathrm{~h}$ using IBM ILOG Cplex Optimization Studio v12.5. According to the results of both models, it is observed that kidney flow in the base models is $2.17 \%$ greater on average compared to the extended one. This was expected as the base model maximizes only the overall kidney flow. On the other hand, when the extended model considers heart and liver flows in addition, it is observed that there are improvements in the flow of these two organs. For instance when the case $\alpha=40 \%$ and $\beta=2 \%$ is examined, the kidney flow decreases around $2.3 \%$, while the heart flow increases approximately $8 \%$ and liver flow increases about 
Table 12 Change observed in the flows in COT-E from COT $(\%)$

\begin{tabular}{|c|c|c|c|c|}
\hline & & Heart & Liver & Kidney \\
\hline \multirow[t]{3}{*}{$\alpha=45 \%$} & $\beta=1 \%$ & $5.69 \%$ & $3.61 \%$ & $-2.68 \%$ \\
\hline & $\beta=2 \%$ & $1.72 \%$ & $1.72 \%$ & $-1.59 \%$ \\
\hline & $\beta=3 \%$ & $10.67 \%$ & $3.61 \%$ & $-2.05 \%$ \\
\hline \multirow[t]{3}{*}{$\alpha=40 \%$} & $\beta=1 \%$ & $4.67 \%$ & $1.09 \%$ & $-2.21 \%$ \\
\hline & $\beta=2 \%$ & $7.98 \%$ & $3.19 \%$ & $-2.31 \%$ \\
\hline & $\beta=3 \%$ & $6.67 \%$ & $3.97 \%$ & $-2.34 \%$ \\
\hline \multirow[t]{3}{*}{$\alpha=35 \%$} & $\beta=1 \%$ & $2.17 \%$ & $3.52 \%$ & $-0.24 \%$ \\
\hline & $\beta=2 \%$ & $6.01 \%$ & $5.95 \%$ & $-2.55 \%$ \\
\hline & $\beta=3 \%$ & $3.17 \%$ & $1.09 \%$ & $-3.54 \%$ \\
\hline
\end{tabular}

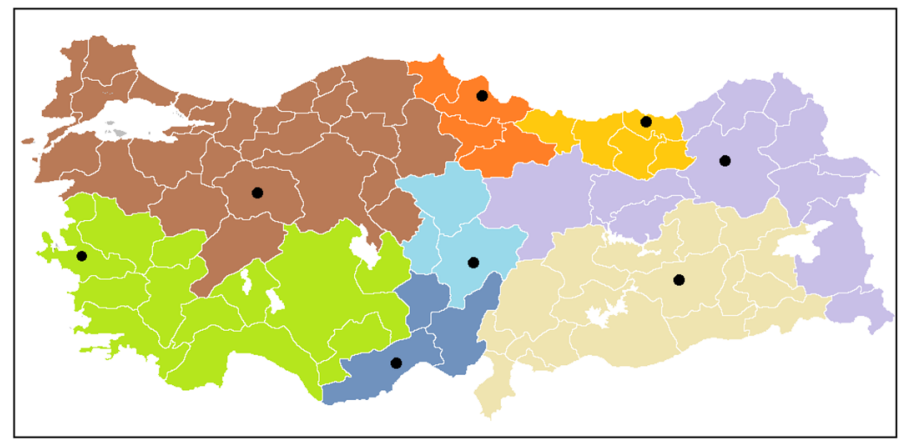

Fig. 6 COT-E for $p=8, \alpha=40 \%$ and $\beta=2 \%$

$3.2 \%$. The remaining instances also demonstrate a very similar structure; significant growths can be obtained in heart and liver flows, while the decreases in kidney flow always remain relatively smaller and at the same level. These results indicate that the COT-E model serves better than the COT model for the individual organ types. The clustering of the $\alpha=40 \%$ and $\beta=2 \%$ instance is illustrated on a map in Figure 6 as an example.

\section{Conclusions}

This study analyzes the organ transportation logistics in hierarchical systems. It is observed that the clustering structure plays a critical role in the matching processes within the hierarchy. Therefore, we have proposed clustering-based models with alternative transportation options to find the optimal clusters for organ transplantation networks. Additionally, we developed a simulation model to analyze the outputs of the deterministic model in an uncertain environment which represents the real-life characteristics of hierarchical systems. We tested the models' performances by using data set of Turkey. 
Our analysis reveals that when the number of RCCs is fixed to 8 in the COT, the maximum travel time from the donor city to the recipient city turns out to be $314 \mathrm{~min}$ (Fig. 5), which satisfies the time bounds for liver (405 min) and kidney (570 min) transplantations. Since the objective of the model is to maximize the total potential organ flow within the regions, the model allocates each city to the RCC whose region maximizes the potential organ flow even if this RCC is not the closest one.

In order to satisfy the time bound of the heart, ground and air transportation must be used together. The current system utilizes 14 helicopters with 9 RCCs, where the proposed solution with $8 \mathrm{RCC}$ regions requires only 5 helicopters. When the number of the RCCs is fixed ( $p=8$ ), transportation times can be decreased by more than $2.5 \mathrm{~h}$ with the proposed solution. Moreover, the objective function value representing potential-weighted intra-regional flow increases with a lower $T$ value.

With the current system, neither liver nor heart transplantations can be performed without air transportation. The proposed solution enables the transportation of livers by terrestrial methods, not only with 8 RCCs but also with 6 . For kidney transportation, the required number of RCC regions is 4 . It is observed that decreasing the number of RCCs increases the number of matches at the same RCC due to the increase in the number of potential donors in the region. When an organ is matched with a recipient from the same RCC, the proposed systems perform better than the current system for all organ types. In the proposed system, the usage of airborne transportation methods is much lower than in the current system; specifically helicopter usage is approximately 16 times lower.

In the extension, all organs are treated as equally important, and it is observed that this increases the overall balance of the network in terms of obtaining higher heart and liver flows while decreasing the kidney flow by a small amount. The average of 9 instances reveals a $5.4 \%$ increase in heart and $3.1 \%$ increase in liver flows, whereas a $2.1 \%$ decrease in kidney flow.

In this study, we have greatly improved Turkey's organ transplantation system. One of the main contributions of this study is to highlight the logistical issues in organ transplantation and guide the decision makers while optimizing these systems. We not only have found optimal clusters for the hierarchical systems, but we have also measured the performance of the deterministically grounded model in an uncertain, more complex problem with a discrete-event simulation model. Our methods improve system performance by maximizing potential-weighted intra-regional flow and highlighting critical cities that define the average transportation time bounds in a cluster. Moreover, by decreasing the transportation time from the donor city to the recipient city, we increase the time available for surgery, which indirectly improves surgery performance, thus increasing the likelihood of a successful transplantation. We hope that this study can be expanded to other countries using a hierarchical organ transplantation system to find optimal clusters that maximize potential intra-regional flow.

Acknowledgements The authors would like to express their gratitude to Dr. Kahveci, who is a member of National Organ and Tissue Transplant Board in Turkey, for his valuable comments and help in granting us access to the Turkish organ transplantation data. 


\section{References}

Alagoz O, Schaefer AJ, Roberts MS (2009) Optimizing organ allocation and acceptance. In: Pardalos P, Romeijn E (eds) Handbook of optimization in medicine. Springer, Berlin, pp 1-24

Araz C, Selim H, Ozkarahan I (2007) A fuzzy multi-objective covering-based vehicle location model for emergency services. Comput Oper Res 34(3):705-726

Beliën J, De Boeck L, Colpaert J, Devesse S, Van Den Bossche F (2012) Optimizing the facility location design of organ transplant centers. Decis Support Syst 54(4):1568-1579

Bertsimas D, Farias VF, Trichakis N (2013) Fairness, efficiency, and flexibility in organ allocation for kidney transplantation. Oper Res 61(1):73-87

Brailsford SC (2005) Overcoming barriers to the implementation of OR simulation models in healthcare. J Clin Investig Med 28:312-315

Bruni ME, Conforti D, Sicilia N, Trotta S (2006) A new organ transplantation location-allocation policy: a case study of Italy. Health Care Manag Sci 9(2):125-142

Chaiwuttisak P, Smith H, Wu Y, Potts C, Sakuldamrongpanich T, Pathomsiri S (2016) Location of low-cost blood collection and distribution centres in Thailand. Oper Res Health Care 9:7-15

Dag A, Topuz K, Oztekin A, Bulur S, Megahed FM (2016) A probabilistic data-driven framework for scoring the preoperative recipient-donor heart transplant survival. Decis Supp Syst 86:1-12

Deffains B, Ythier JM (2010) Optimal production of transplant care services. J Pub Econ 94(9-10):638-653

Demirci MC, Schaefer AJ, Romeijn HE, Roberts MS (2012) An exact method for balancing efficiency and equity in the liver allocation hierarchy. INFORMS J Comput 24(2):260-275

Drekic S, Stanford DA, Woolford DG, McAlister VC (2015) A model for deceased-donor transplant queue waiting times. Queueing Syst 79(1):87-115

Friedewald JJ, Samana CJ, Kasiske BL, Israni AK, Stewart D, Cherikh W, Formina RN (2013) The kidney allocation system. Surg Clin N Am 93:1395-1406

Genc R (2008) The logistics management and coordination in procurement phase of organ transplantation. Tohoku J Exp Med 216(4):287-296

Gentry S, Chow E, Massie A, Segev D (2015) Gerrymandering for justice: redistricting U.S. liver allocation. Interfaces 45(5):462-480

Harper AM, Taranto SE, Edwards EB, Daily OP (2000) An update on a successful simulation project: The UNOS liver allocation model, In: Proceedings of the 2000 Winter Simulation Conference, Piscataway, NJ (Institute of Electrical and Electronics Engineers, NJ, 2000)

Human organ transplantation. World Health Organization. 15 June, (2016). http://www.who.int/ transplantation/organ/en/

Jabbarzadeh A, Fahimnia B, Seuring S (2014) Dynamic supply chain network design for the supply of blood in disasters: a robust model with real world application. Transp Res Part E 70:225-244

Kong N, Schaefer AJ, Hunsaker B, Roberts MS (2010) Maximizing the efficiency of the U.S. liver allocation system through region design. Manag Sci 56:2111-2122

Liberatore M, Nydick R (2008) The analytic hierarchy process in medical and healthcare decision making: a literature review. Eur J Oper Res 189(1):194-207

Matching Organs (2012) Transplant living. United Network for Organ Sharing. http://www.transplantliving. org/before-the-transplant/about-organ-allocation/matching-organs/

Misiunas N, Oztekin A, Chen Y, Chandra K (2016) DEANN: a healthcare analytic methodology of data envelopment analysis and artificial neural networks for the prediction of organ recipient functional status. Omega 58:46-54

Mousazadeh M, Torabi SA, Zahiri B (2015) A robust possibilistic programming approach for pharmaceutical supply chain network design. Comput Chem Eng 82:115-128

Nagurney A, Masoumi AH (2012) Supply chain network design of a sustainable blood banking system. Sustainable supply chains. Springer, New York, pp 49-72

OPTN (2016) How organ allocation works. Organ Procurement and Transplantation Network. https://optn. transplant.hrsa.gov/learn/about-transplantation/how-organ-allocation-works/

Organ Mafia (2012) Aksiyon. Aksiyon Magazine. http://www.aksiyon.com.tr/aksiyon/haber-3059-26organ-mafyasi.html

Rais A, Viana A (2011) Operations research in healthcare: a survey. Int Trans Oper Res 18(1):1-31

Ratcliffe J, Young T, Buxton M, Eldabi T, Paul R, Burroughs A, Papatheodoridis G, Rolles K (2001) A simulation modeling approach to evaluating alternative policies for the management of the waiting list for liver transplantation. Health Care Manag Sci 4:117-124. Kluwer Academic Publishers 
Recent Data (2016) Department of Organ and Tissue Transplantation and Dialysis Services. Re-public of Turkey Ministry of Health. https://organ.saglik.gov.tr/web

Republic of Turkey Ministry of Health (2012) Department of Organ and Tissue Transplantation Services. National Organ Transplantation Database (Ulusal Organ Nakli Bilgi Sistemi). Retrieved Nov 2012

Sahin G, Sural H, Meral S (2007) Locational analysis for regionalization of Turkish Red Crescent blood services. Comput Oper Res 34(3):692-704

Sha Y, Huang J (2012) The multi-period location-allocation problem of engineering emergency blood supply systems. Syst Eng Procedia 5:21-28

Shariff SSR, Moin NH, Omar M (2012) Location allocation modeling for health-care facility planning in Malaysia. Comput Ind Eng 62:1000-1010

Shechter SM, Bryce C, Alagoz O, Kreke JE, Stahl JE, Schaefer AJ, Angus D, Roberts M (2005) A clinically based discrete event simulation of end-stage liver disease and the organ allocation process. Med Decis Mak 25(2):199-209

Shirabe T (2009) Districting modeling with exact contiguity constraints. Environ Plan B Plan Des 36(6):1053-1066

Smith HK, Harper PR, Potts CN (2013) Bicriteria efficiency/equity hierarchical location models for public service application. J Oper Res Soc 64(4):500-512

Smith JM, Biggings SW, Haselby DG, Kim WR, Wedd J, Lamb K, Thompson B, Segev DL, Gustafson S, Kandaswamy R, Stock PG, Matas AJ, Samana CJ, Sleeman EF, Stewart D, Harper A, Edwards E, Snyder JJ, Kasiske BL, Israni AK (2012) Kidney, pancreas and liver allocation and distribution in the United States. Am J Transpl 12:3191-3212

Stahl JE, Kong N, Shechter SM, Schaefer AJ, Roberts MS (2005) A methodological framework for optimally reorganizing liver transplant regions. Med Decis Mak 25(1):35-46

$\mathrm{Su}$ X, Zenios SA (2005) Patient choice in kidney allocation: a sequential stochastic assignment model. Oper Res 53(3):443-455

Syam SS, Cote MJ (2010) A location-allocation model for service providers with application to not-forprofit health care organizations. Omega 38(3-4):157-166

Syam SS, Cote MJ (2012) A comprehensive location-allocation method for specialized healthcare services. Oper Res Health Care 1(4):73-83

Taranto SE, Harper AM, Edwards EB, Rosendale JD, McBride MA, Daily OP, Murphy D, Poos B, Reust JS, Schmeiser BW (2000) Developing a national allocation model for cadaveric kidneys. In: Fishwick PA, Kang K, Joines IA, Barton RR (eds) society of computer simulation international 32nd winter simulation conference, Orlando, pp 1971-1977

Thompson D, Waisanen L, Wolfe R, Merion R, McCullough K, Rodgers A (2004) Simulating the allocation of organs for transplantation. Health Care Manag Sci 7(4):331-338

Treatments and Procedures (2012) Organ Donation and Transplantation. Cleveland Clinic. http://my. clevelandclinic.org/services/organ_transplant/hic_organ_donation_and_transplantation.aspx

Uehlinger NB, Beyeler F, Weiss J, Marti HP, Immer F (2010) Organ transplantation in Switzerland: impact of the new transplant law on cold ischemia time and organ transports. Swiss Med Wkly 140:222-227

UNOS (2018) Data. United Network for Organ Sharing. https://unos.org/data/

Zahiri B, Pishvaee MS (2017) Blood supply chain network design considering blood group compatibility under uncertainty. Int J Prod Res 55(7):2013-2033

Zahiri B, Mousazadeh M, Bozorgi-Amiri A (2014a) A robust stochastic programming approach for blood collection and distribution network design. Int J Res Ind Eng 3(2):1-11

Zahiri B, Tavakkoli-Moghaddam R, Pishvaee MS (2014b) A robust possibilistic programming approach to multi-period location-allocation of organ transplant centers under uncertainty. Comput Ind Eng 74:139-148

Zahiri B, Tavakkoli-Moghaddam R, Mohammadi M, Jula P (2014c) Multi-objective design of an organ transplant network under uncertainty. Transp Res Part E Logist Transp Rev 72:101-124

Zahiri B, Torabi SA, Mousazadeh M, Mansouri SA (2015) Blood collection management: methodology and application. Appl Math Modell 39:7680-7696 